\title{
EL PROCESO DE INTERNALIZACIÓN EN EL DESARROLLO CIENTIFICO*
}

ANA ROSA PEREZ RANSANZ Instituto de Investigaciones Filos6ficas, UNAM

Shapere intenta dar cuenta de la racionalidad y de la objetividad de la ciencia en una forma que resulte compatible con la idea de que la ciencia no funciona sin presupuestos o no hay ciencia libre de presupuestos. Uno de los principales méritos del trabajo de Shapere consiste, justamente, en emprender un análisis detallado de las distintas formas en que los presupuestos operan en la ciencia, e incluso pretende establecer ciertos límites en cuanto a la naturaleza y al funcionamiento de dichos presupuestos. Así, refiriéndose a la objetividad, Shapere nos dice: "la objetividad de la ciencia puede sostenerse a pesar de la necesaria utilización de creencias presupuestas (creencias del background), siempre y cuando podamos distinguir ciertas creencias como antecedentes legítimos de otras que no lo son, y a condición de que la base para hacer tal distinción pueda, ella misma, ser legitimizada".'

Esta afirmación plantea dos tipos de problemas: uno con respecto a la caracterización de los presupuestos que son legítimos, y otro con respecto a los criterios mismos que se utilizan al hacer esta caracterización. El primer tipo de problemas consiste en acotar aquellas creencias previas cuya utilización es legítima en el trabajo científico, en aclarar los distintos modos en que estas creencias son utilizadas en la búsqueda y adquisición del conocimiento, y además en dar cuenta de cómo se justifican estos usos. Las posibles res-

- Trabajo presentado en el VII Simposio de Filosofía del Instituto de Investigariones Filosóficas, UNAM, agosto de 1986.

I D. Shapere, "Observation, Experiment, and Interpretation" (sin publicar), p. 12 (subrayado mío). 
puestas a estas cuestiones se darán obviamente en función de los criterios que se adopten, lo cual nos lleva al segundo tipo de problemas: hacer explícitos estos criterios, analizar cómo se han desarrollado históricamente y mostrar si es posible o no legitimar los criterios mismos sin caer en una circularidad viciosa.

El objetivo de este trabajo es analizar la respuesta que ofrece Shapere a estos dos grupos de problemas. Si bien las tesis involucradas en su respuesta constituyen el sustrato de sus ideas acerca de la objetividad y la racionalidad científicas, no me ocuparé, por el momento, de discutir estas últimas.

La clave para responder a los problemas planteados radica, según Shapere, en el simple hecho de que no cualquier creencia puede funcionar como creencia presupuesta (background belief) en la actividad científica. Las creencias presupuestas son aquellas que juegan un papel definido, específico, en la conformación de la actividad científica y en su interpretación teórica. ${ }^{2}$ Son creencias que han satisfecho severos requisitos y han ganado por ello el derecho a ser consideradas como información legítimamente utilizable, información que de hecho es usada tanto en la concepción como en la interpretación de los datos, de los problemas, de los métodos, de los criterios de solución de problemas, e incluso de los objetivos mismos de la investigación. Los requisitos más importantes que debe satisfacer una creencia para tener el status de información legítimamente utilizable son los siguientes: (1) haber sido exitosa, (2) estar libre de dudas específicas y apremiantes, y (3) ser claramente relevante para el objeto de estudio o problema en cuestión. ${ }^{3}$

El problema ahora es entender las ideas de "éxito", "ausencia de dudas específicas" y "relevancia". Al rastrear el origen de la manera actual de entender estas nociones, Shapere lo encuentra en los siglos XVII y XVIII,

${ }^{2}$ Cfr. D. Shapere, "Ojectivity, Rationality, and Scientific Change", por aparecer en $P$. Kitcher y P. Asquith (eds.). PSA 84, Vol. II, 1986, p. 4.

${ }^{3}$ Cfr. ibid. 
época en que se difunde y se instaura una cierta manera peculiar de examinar la naturaleza. Este enfoque, que Shapere denomina "enfoque por parcelas" (piecemeal approach), viene a reemplazar el tipo de acercamiento que dominaba desde la época de los griegos y que tuvo una amplia difusión en la Edad Media. El viejo enfoque tenía un carácter fundamentalmente holista en el sentido de que pretendía, por ejemplo, explicar la naturaleza del cambio o de la sustancia en general, o determinar las condiciones necesarias del conocimiento en general. ${ }^{4}$ Por cierto, Shapere señala que esta manera de acercarse a los problemas no murió totalmente; prueba de ello son los escritos de una buena cantidad de filósofos actuales. ${ }^{5}$ El enfoque por parcelas difiere del enfoque holista básicamente en cuanto a la metodología empleada. Bajo este enfoque, se examina una cierta región de la experiencia, aislándola del resto. Shapere llama "dominios" a estas áreas de investigación específicas y aisladas, y las caracteriza de la siguiente manera: un dominio es una asociación de ítems, asociación que constituye un área de investigación; los dominios tienen las siguientes características: "(1) la asociación está basada en alguna relación (o supuesta relación); (2) hay algo problemático acerca del cuerpo de información así relacionado; (3) ese problema es un problema importantè o relevante". 6

Shapere habla de "ítems" para utilizar un término neutro con respecto a las distintas categorías ontológicas, como por ejemplo "objeto", "evento", "propiedad", etc. Y además porque estas categorías están sujetas a revisión a la luz de lo que vamos aprendiendo en nuestro estudio de la naturaleza.?

La existencia de dominios trae consigo la posibilidad de clasificar sistemáticamente los distintos tipos de problemas que pueden surgir en este enfoque por parcelas. Dentro de

${ }^{4}$ Cfr. ibid., p. 5.

${ }^{5}$ Cfr. ibid., p. 20.

${ }^{6}$ Ibid., p. 5.

${ }^{7}$ Cfr. ibid., p. 21. 
esta clasificación existen dos tipos de problemas que resultan centrales para entender las nociones de "éxito" y de "ausencia de dudas específicas". El primer tipo se refiere a la cuestión de si una teoría o explicación propuesta para un dominio da efectivamente cuenta o no de ese dominio, y, si lo hace, hasta qué punto. Shapere llama a éstos problemas del éxito teórico. El segundo tipo se refiere a problemas de adecuación, dentro de los cuales tenemos los problemas de incorrección, como por ejemplo el de que una teoría pueda encerrar inconsistencias, o el de que haya razones para suponer que una explicación es no-realista al tratar su dominio (es decir, que conste solamente de dispositivos conceptuales, como idealizaciones, ficciones convenientes, simplificaciones, etc.), o, finalmente, el de que la explicación de un dominio particular no sea compatible con teorías de otros dominios, cuando existen razones para suponer que debería serlo. ${ }^{\sharp}$

Entonces, una teoría (o conjunto de creencias) es exitosa en la medida en que da cuenta completamente de todos los items de su dominio. Y una teoría (o conjunto de creencias) está libre de dudas específicas cuando, por el momento, no tiene problemas de incorrección, es decir, cuando es consistente, cuando es considerada una explicación realista y cuando es compatible con las teorías con las que se supone que debe serlo. Estas dos características son independientes, pues una teoría puede tener problemas de éxito teórico y no tener problemas de incorrección, y viceversa (puede ser exitosa con respecto a su dominio y, sin embargo, haber razones para pensar que es incorrecta). ${ }^{9}$

Con los anteriores elementos ya nos podemos acercar a una de las tesis centrales de Shapere: "Aquello por lo que la ciencia se esfuerza consiste en admitir como creencias previas legítimamente utilizables únicamente aquellas teorías previas que se han mostrado exitosas con respecto a sus pro-

" D. Shapere, "Reason and the Search for Truth" (manuscrito), pp. 6-7.

${ }^{9}$ Cfr. ibid., p. 8. 
pios dominios. . y libres de dudas específicas. . " 10 Por tanto, para Shapere el conocimiento está constituido por aquellas creencias que, en cada momento histórico, satisfacen los requisitos de éxito y ausencia de dudas con relación a su corrección. Estas son las creencias que en la ciencia cuentan o funcionan como razones, y a ellas apela la ciencia en sus deliberaciones.

Debemos decir que Shapere reconoce que la ciencia emplea de hecho proposiciones o creencias que no cumplen estos rigurosos criterios. En el trabajo científico se proponen continuamente hipótesis o conjeturas no sólo para resolver problemas existentes sino también para introducir nuevos problemas y construir nuevas hipótesis. Esto es, las hipótesis o conjeturas juegan los mismos papeles que juegan las creencias que son consideradas como conocimiento, y ello con el fin de extender este conocimiento. Frente a esto Shapere no se preocupa, pues afirma que su idea central se sigue sosteniendo, ya que "detrás de esas mismas hipótesis y de su utilización en llevar más lejos el trabajo de la ciencia, está un cuerpo (background) de creencias que han probado satisfacer esos estándares (o criterios) en un grado muy alto, y en términos de las cuales las hipótesis han sido formuladas y son aplicadas"."

También acepta Shapere que existe otro tipo de situaciones en las cuales la ciencia no siempre parte de o se apoya en creencias legítimas. Este caso se presenta cuando la información o conocimiento establecido resulta insuficiente como base para seleccionar y describir los objetos de estudio, para plantear problemas, para diseñar métodos de solución, para perfilar lo que sería una solución adecuada, etc., etc. La realización de todas estas tareas, cuando el conocimiento disponible es insuficiente, requiere entonces la utilización de creencias o consideraciones que son externas a este conocimiento; y en la concepción de Shapere, como

${ }^{10}$ D. Shapere, "Objectivity, Rationality, and Scientific Change", p. 9.

11 lbid., p.11. 
vimos, estas consideraciones externas no pueden tener el status de razones.

Frente a estas situaciones en que necesariamente se ponen en juego presupuestos no legítimos, Shapere adopta la vía de minimizarlas y restarles importancia. Esto queda claro, en mi opinión, cuando afirma que "sin embargo, tales casos tienden a encontrarse en lo que claramente son casos históricos, no sofisticados (y usualmente tempranos)" ${ }^{12}$ Más adelante retomaré este punto en conexión con otras tesis de Shapere.

La respuesta de Shapere frente a este tipo de situaciones apunta claramente a otra de sus tesis centrales. Según él, es imposible negar que la ciencia se ha vuelto cada vez más capaz de apoyarse en el bagaje acumulado de conocimientos, y que este último se ha vuelto una base cada vez más suficiente para la realización de las tareas científicas. Esta tesis que postula la tendencia de la ciencia a ser autosuficiente o autónoma, es, según Shapere, un resultado de análisis históricos. Es la constatación de una cuestión de hecho o, más precisamente, la constatación del curso que ha seguido la ciencia en su desarrollo histórico.

Shapere trata de explicar esta tendencia a la autonomía analizando la dinámica del cambio científico. Este análisis lo lleva a la conclusión de que la ciencia se desarrolla por medio de un proceso de internalización. Para entender en qué consiste este proceso de internalización tenemos que considerar ahora la respuesta que da Shapere al segundo grupo de problemas que planteamos al principio de este trabajo: los problemas concernientes a los criterios mismos con base en los cuales se decide acerca de la legitimidad y el funcionamiento de los presupuestos en la ciencia. Ya vimos que para que una creencia sirva como presupuesto científico legítimo debe satisfacer los criterios de éxito y ausencia de dudas específicas. Debemos preguntarnos ahora por la naturaleza y la legitimidad de esos mismos criterios.

12 Ibid., p. 12. 
Tomemos, por ejemplo, el criterio del éxito formulado de la siguiente manera: La teoría de un dominio debe dar cuenta de una manera completa y satisfactoria de todos los ítems de ese dominio. Shapere encuentra que el criterio mismo es producto de un proceso histórico y que ha sufrido alteraciones como resultado del curso de la investigación. ${ }^{13}$ Incluso la adopción misma del criterio, bajo esa formulación, no fue posible sino hasta el siglo XVII, cuando se afianza el enfoque por parcelas (piecemeal approach). Este enfoque reemplaza a otros enfoques que manejaban concepciones distintas del éxito.

Uno de los aspectos más interesantes de las tesis de Shapere acerca de criterios como el del éxito se muestra cuando afirma que el criterio, tal como él lo ha formulado, no funciona en realidad como un criterio, es decir, en el sentido de proporcionar instrucciones específicas sobre lo que el científico debe hacer ante una situación problemática dada. Esto lleva a Shapere a hacer una distinción entre "esquemas de criterios" o "criterios-esquema", por una parte, y "especificaciones" de esos esquemas o "criterios específicos", por otra, siendo estos últimos los que en la práctica funcionan como criterios en sentido estricto. ${ }^{14}$

En el caso del éxito, la formulación general que ofrece Shapere viene a ser el esquema del criterio. Pero ¿cómo entender los conceptos que entran en una formulación general? Por ejemplo: qué cuenta como ítem en una situación dada, cómo deben describirse esos ítems, qué califica como explicación satisfactoria y completa, etc. La especificación de estos conceptos estará dada por criterios - que son especificaciones de los esquemas - que la ciencia ha desarrollado, es decir, por la información relevante disponible en cada época científica particular.

Ahora bien, dado que las mismas creencias que alcanzan el status de conocimiento cambian, se tiene entonces que las especificaciones de los criterios-esquema cambian también.

${ }^{13}$ Cfr. ibid., p. 14.

14 Cfr. D. Shapere, "Reason and the Search for Truth", p. 16. 
Esta sujeción de los criterios específicos al cambio y a la corrección es el resultado de la remodelación de las creencias aceptadas, aceptación que a su vez resulta de la aplicación de esos criterios específicos. Existe entonces una interacción dinámica continua entre los criterios y el cuerpo de creencias que se acepta con base en esos criterios. ${ }^{15}$ Es mediante esta dinámica que los criterios se han despojado, de hecho, de todo carácter trascendente - trascendente con relación a la ciencia - y se han hecho parte del proceso científico mismo, es decir, han sido internalizados.

Así, aun cuando una ciencia al comenzar a desarrollarse, o en ciertas etapas de su desarrollo, recurra a creencias y criterios externos, el proceso de internalización, tarde o temprano, los sujetará a los procedimientos de revisión que ellos mismos han ayudado a establecer. Es por medio de este proceso de internalización que todos los aspectos de la actividad científica se llegan a someter a contrastación.

Shapere señala, con toda razón, que si los criterios, como los del éxito y la ausencia de dudas específicas, fueran irrevocablemente trascendentes a los procesos y resultados científicos, entonces esos criterios requerirían una justificación o legitimación que no podría venir de la ciencia misma. Pero la historia de la ciencia nos muestra lo contrario: el carácter autocorrectivo del proceso nos hace ver cómo, de hecho, los criterios han sido revisados, refinados o abandonados, a la luz de los descubrimientos que estos mismos criterios han posibilitado.

Cabe aclarar que para Shapere el proceso de internalización no es un proceso necesario en ningún sentido; es un proceso que ha ocurrido contingentemente. Sin embargo, el hecho de que haya sido así nos da bases para postularlo como una meta que hay que seguir: la ciencia no sólo ha intentado, sino que debe seguir intentando hacer explícitos y someter a prueba sus presupuestos más fundamentales, tanto los presupuestos sustantivos como los presupuestos metodológicos.

15 Cfr. D. Shapere, "Objectivity, Rationality, and Scientific Change", p. 15. 
Una vez delineada la concepción de Shapere acerca del proceso de desarrollo del conocimiento científico, y del papel que en él juegan los presupuestos, quisiera hacer algunas observaciones.

Considero que el proceso de internalización, tal como lo describe Shapere, necesita un complemento. Es decir, el desarrollo del conocimiento científico no queda completamente descrito si se concibe como un puro proceso de internalización. La otra cara de la moneda, si aceptamos este proceso, tendría que consistir en un proceso de eliminación o de externalización. Tomando como ejemplo un caso que utiliza el mismo Shapere, podemos ver cómo en el trabajo de Laplace se eliminan las hipótesis teológicas inmersas en la teoría de Newton. Esto es, el desarrollo del conocimiento también permite desechar hipótesis o creencias que, en un momento dado, forman parte constitutiva de las teorías y juegan un papel importante en el interior de ellas. Creencias que en un cierto momento funcionan como factores internos pasan a ser consideradas como factores externos. Es posible que esta contraparte del proceso pudiera ser inferida a partir de la internalización; sin embargo, me parece necesario hacerla explícita para lograr una mejor comprensión del desarrollo científico desde el punto de vista adoptado por el mismo Shapere.

Otra cuestión relacionada con el proceso de internalización es la siguiente: Shapere afirma que existe abundante evidencia histórica de que este proceso efectivamente tiene y ha tenido lugar. Sin embargo, la "evidencia" utilizada por Shapere proviene, en su mayor parte, de la física, y principalmente de la física desarrollada en este siglo a partir de los años 30. En mi opinión Shapere extrapola, en forma peligrosa y cuestionable, lo que ocurre en una ciencia "altamente sofisticada" al resto de las ciencias. Quizá esto se deba a que implícitamente Shapere está a favor de un monismo metodológico que toma como modelo de cientificidad a la física contemporánea. Esta hipótesis sobre la simpatía de Shapere por un monismo metodológico se ve reforzada por el hecho de que el autor constantemente formula sus tesis 
centrales en términos de "la ciencia", sin matizar estas tesis de acuerdo con lo que ocurre o ha ocurrido en las distintas ciencias particulares.

Por otro lado, la crítica que hace Shapere a los defensores del relativismo también nos da elementos a favor de esta hipótesis. El relativismo criticado es el que afirma que la ciencia, en cualquier etapa de su historia, emplea al interpretar la experiencia y la naturaleza, presupuestos que son, en última instancia, arbitrarios; es decir, niega la posibilidad de que haya presupuestos legítimamente utilizables (en el sentido de Shapere). De aquí que para estos relativistas los procedimientos científicos no merezcan que se les califique de objetivos ni de racionales, y que los resultados de la investigación científica no merezcan el título de conocimiento. ${ }^{16}$

Shapere afirma que esta concepción está equivocada porque se basa en una generalización de lo que ha ocurrido en episodios tempranos de la historia de la ciencia. Sin embargo, tal parece que Shapere, a su vez, basa su concepción en una generalización de lo que ha ocurrido en episodios tardíos de las ciencias altamente desarrolladas. Digo esto porque, además de las razones apuntadas, en su crítica al relativismo parece negar que la necesidad de recurrir a creencias no legítimas (según sus criterios), sea un fenómeno que se sigue presentando y ocurre con mucha frecuencia en una gran cantidad de campos de la investigación científica (los campos que están situados fuera de la física y de ciertas parcelas de la química y de la biología). ¿O acaso diría Shapere que los trabajos desarrollados en esos campos se encuentran en un estadio inmaduro o precientífico? ¿Qué status de cientificidad otorgaría, por ejemplo, a las ciencias sociales?

Shapere exhorta a los filósofos a que adopten y apliquen el "piecemeal approach", pero él mismo no lo hace. Las conclusiones de sus análisis serían altamente plausibles, e incluso válidas, siempre y cuando delimitara el dominio o campo de aplicación de estas conclusiones, que es el campo

${ }^{16}$ Cfr. ibid., pp. 2.3 . 
de las "ciencias sofisticadas", como él mismo las llama. Esto es, si Shapere siguiera su propio consejo, dejaría de hacer afirmaciones sobre la ciencia en general, y sus conclusiones tendrían validez en la medida en que restringiera su dominio de aplicación.

Paso a otra observación. La teoría de la ciencia que desarrolla Shapere es una teoría basada en la historia de la ciencia, en el sentido de utilizarla ampliamente. Shapere está presuponiendo en sus análisis que contamos con una información o conocimiento histórico adecuado, es decir, que existe un cúmulo de creencias históricas legítimamente utilizables. Así, en un artículo sobre las relaciones entre historia y filosofía de la ciencia, Shapere afirma que la historia de la ciencia ha sufrido, en las últimas décadas, una profunda transformación en el sentido de que sus estándares de investigación "se han vuelto mucho más sofisticados". ${ }^{17}$ Estas palabras de Shapere significan, como hemos visto, que la historia de la ciencia ha desarrollado criterios autónomos y autosuficientes. Sin embargo, Shapere no analiza más a fondo esta cuestión, e incluso afirma que "no necesitamos dar una explicación completa y sistemática de los criterios de la investigación histórica que muestra su objetividad... para reconocer que tal investigación es posible" ${ }^{18}$

Ahora bien, si se asume - como lo hace Shapere- que la investigación histórica no proporciona datos neutros, libres de teoría, entonces la confianza en determinadas creencias históricas tendría que estar justificada por una teoría del conocimiento histórico que permitiera, por así decirlo, seleccionar la buena historia de la mala historia. Por tanto, a diferencia de Shapere, considero que una teoría de la ciencia o del conocimiento que se apoye fuertemente en análisis históricos, está obligada a dar una teoría o explicación sistemática del conocimiento histórico mismo.

17 D. Shapere, "What Can the Theory of Knowledge Learn from the History of Knowledge?", en D. Shapere, Reason and the Search for Knowledge, Boston Studies in the Philosophy of Science, vol. 78, Reidel Publishing Company, Dordrecht, Holland, 1984.

18 Ibid., p. 199. 
Shapere afirma que su interés está en "la ciencia" y no en "los científicos", porque le interesan las razones y, por lo tanto, las relaciones entre las creencias, y entre las creencias y los criterios. ${ }^{19}$ Es decir, él se ocupa del contenido de la ciencia de una época determinada y de sus implicaciones, y no se ocupa de los científicos porque es posible que ese contenido "no haya sido adecuadamente entendido (o no entendido en absoluto) por los científicos individuales de esa época, o incluso por ninguno de ellos". ${ }^{20}$

Esta afirmación no nos sorprendería en modo alguno si viniera de autores como Reichenbach o Carnap, quienes tenían como objetivo realizar reconstrucciones lógicas del conocimiento científico y no reconstrucciones históricas. Pero resulta difícil de entender viniendo de alguien que pretende analizar el proceso científico tal como se ha dado de hecho, y que además se apoya tan fuertemente en las fuentes y documentos históricos. Jugando el papel de abogado del diablo, le preguntaría a Shapere si es posible hacer una reconstrucción racional, de corte histórico, del desarrollo científico, al margen de las creencias efectivamente sustentadas por los científicos de cada época. Un crítico de Shapere podría afirmar que para entender la estructura racional de la investigación científica es necesario ubicarla en su contexto histórico, el cual incluye las creencias y puntos de vista de los investigadores que de hecho producen, evalúan y aceptan o rechazan las teorías. Además, las relaciones conceptuales entre teorías sucesivas se pueden examinar sólo gracias a que esas teorías han sido, en algún momento, aceptadas por la comunidad pertinente. El hecho de que se llegue a una teoría por una vía racional no es suficiente para que forme parte del cuerpo de la ciencia. Esto requiere de una decisión del grupo de investigadores que hacen, que construyen, la disciplina en cuestión. Por tanto, una comprensión adecuada y completa del desarrollo de las distintas ciencias tiene que tomar en cuenta las creencias de

19 Cfr. D. Shapere, "Objectivity, Rationality, and Scientific Change", nota 1, p. 20.

20 lbid. 
hecho sustentadas, sean éstas internas o externas, científicas o no.

Quisiera terminar comentando uno de los aspectos más estimulantes del pensamiento de Shapere, que es su optimismo. Este optimismo consiste en considerar que la razón, en principio, no tiene límites; que el conocimiento, a pesar de no tener garantía o fundamento alguno ha alcanzado niveles de comprensión que no podrían haber sido anticipados. El modelo que propone para el desarrollo del conocimiento - a través del proceso de internalización- es un modelo optimista, pues refleja su idea de una razón que constantemente expande sus horizontes, haciéndose cada vez más autosuficiente. Shapere sostiene que todos los límites que los filósofos han tratado de imponer al conocimiento han sido, de hecho, traspasados por la ciencia. Y si bien es posible que algún día encontráramos que nuestro conocimiento estaba equivocado, no por eso dejaría de asombrarnos la excelencia de los resultados alcanzados. Es justamente en este asombro donde encontramos el leit motiv del pensamiento de Shapere. 